\title{
Joint Interference Alignment And Probabilistic Caching in MIMO Small-Cell Networks
}

\author{
Wei Liu, Senior Member, IEEE, Lingbing Li, Libin Jiao, Haifeng Dai and Gan Zheng, Fellow, IEEE
}

\begin{abstract}
Cache-enabled small base stations (SBS) are capable of relieving the heavy burden of the backhaul link and reducing the transmission latency. The hit probability depends on the coverage probability and caching placement probabilities. However, the interference in the small-cell networks may significantly degrade the coverage probability. In this paper, for MIMO small-cell networks consisting of SBS and users, where both of them are equipped with multiple antennas, a joint interference alignment (IA) and probabilistic caching (JIA-ProbC) scheme is proposed. Using tools from stochastic geometry, the $K$-th order Voronoi cells are constructed to form clusters, where $K$ SBSs cooperatively serve users within each of the $K$-th order Voronoi cells. Then, the IA scheme for MIMO interference channel (IC) is employed to cancel the intra-cluster interference within each $K$-th order Voronoi cell. By exploiting the advantage of multiples antennas at users, the IA scheme can simultaneously support more users interference-free than both the zero forcing (ZF) based interference cancellation scheme for MISO systems and SISO systems without interference management, as more interference can be canceled. Furthermore, the coverage probability is analytically approximated by the a closed-form expression. Moreover, the optimal caching placement probability is analytically derived. Numerical Simulation results show that the proposed JIA-ProbC can significantly outperform the existing joint ZF and probabilistic caching (JZF-ProbC) scheme for MISO systems and SISO probabilistic caching (SISO-ProbC) scheme as well as the joint IA and most popular caching (JIAMPC) caching scheme.
\end{abstract}

Index Terms-Probabilistic caching, Small-cell networks, MIMO, Interference alignment, $K$-th Vonoroi cells, Stochastic geometry

\section{INTRODUCTION}

The demand of communication content-oriented services has been ever increasing [1]-[4]. A large number of popular

W. Liu and L. Li are with the State Key Lab of ISN, Xidian University, 710071, Xi'an, Shaanxi, P. R. China. W. Liu is also with the Science and Technology on Communication Networks Laboratory, the 54th Research Institute of China Electronics Technology Group Corporation, 050002, Shijiazhuang, Hebei, P. R. China. Email:liuweixd@mail.xidian.edu.cn, 1b_li@stu.xidian.edu.cn.

L. Jiao is with the Science and Technology on Communication Networks Laboratory, the 54th Research Institute of China Electronics Technology Group Corporation, 050002, Shijiazhuang, Hebei, P. R. China. Email:jiaolb_sjz@163.com.

H. Dai is with the Chengdu Yaguang Electronics Co., Ltd., 610051, Chengdu, Sichuan, P. R. China. Email:dhf760917@126.com.

G. Zheng is with the Wolfson School of Mechanical, Electrical, and Manufacturing Engineering, Loughborough University, Loughborough LE11 3TU, U.K. Email:g.zheng @lboro.ac.uk.

The financial support of the National Natural Science Foundation of China (61871452), the Royal Societys International Exchanges Scheme under Grant IEC/NSFC/181395 and of the UK Engineering and Physical Sciences Research Council (EPSRC) under the grant EP/N007840/1 are gratefully acknowledged.

Manuscript received XXX, XX, 2015; revised XXX, XX, 2015. contents could be frequently requested by numerous users over a period of time [3], where the same content is repeatedly transmitted, which increases the burden of the backhaul link and the transmission latency [2], [3]. In order to relieve the heavy burden of the backhaul link and reduce the transmission latency, cache-enabled base stations (BSs) have been proposed as a promising solution, where the popular contents are cached at BSs [2], [4]. In this case, BSs need not to download the requested contents from the core network and users can obtain the requested content from BSs directly, which relieves the burden of the backhaul link and reduces the transmission latency [2].

The content caching strategy plays the critical role for benefitting from the cache-enabled BSs [2]. The content caching strategies can be roughly divided into deterministic caching [5]-[7] and probabilistic caching [8]-[12]. For the deterministic caching, every BS caches popular contents according to the popularity, for example the most popular caching (MPC) caching strategy, where every BS only caches the most popular contents until the cache space is full [6]. For the probabilistic caching, every BS independently caches each content according to the corresponding placement probability [8]-[10]. Caching strategies have attracted intensive research interests [5]-[12].

Using tools from stochastic geometry, the performance of the networks is analyzed, where the network nodes, BSs and mobile users, are typically modeled as independent homogeneous Poisson point processes (HPPPs) in a 2-dimensional plane [8]. Furthermore, the hit probability, which means that the BS caches the requested content and successfully transmits the content to the user, is the main metric of networks to analyze the performance [8].

For the single-tier network, BSs are modeled as HPPP, and the hit probability is maximized by optimizing caching placement probabilities in [7]-[9], [11]-[13]. For single-tier small cell networks, the hit probability was maximized in two different kinds of networks architectures, i.e, the alwayson architecture and the dynamic on-off architecture [9]. It is shown that in the always-on architecture the optimal caching placement probabilities only depend on the content request probabilities, while in the dynamic onoff architecture they are also related to the mobile user-to-SBS intensity ratio [9]. For single-tier small cell networks, one cooperative caching strategy was proposed by allocating some part of each SBS's cache space to cache the most popular files, while the remaining part is used to cache the different partition of the less popular files [7]. The hit probability and energy efficiency are maximized, respectively, by optimizing the proportion of the cache space 
caching the most popular files [7]. Furthermore, a probabilistic caching scheme was proposed by exploiting the channel selection diversity that one file can be cached at several helpers and be transmitted by selecting the helper which can provide the largest channel power gain [12]. For small cell networks, one cooperative caching scheme was proposed, where all caching helpers caching the same requested content jointly transmit the file to the user to improve the received signal-to-interferenceplus-noise ratio (SINR) within the cooperative region [13]. Furthermore, a tradeoff between cooperative gain and the number of different files cached was investigated to maximize the hit probability [13]. For multicasting homogeneous networks where the BS can simultaneously serve multiple users who request the different files by bandwidth resource allocation, the hit probability is maximized by jointly optimizing the bandwidth allocation and the number of the users served simultaneously [11].

For the two-tier heterogeneous network (HetNet) consisting of edge servers and caching helpers [14], one caching scheme was proposed where caching helpers can only cache the files which are not cached at the edge servers, if they are covered by edge servers, while the other caching helpers can cache arbitrarily files. Furthermore, the hit probability is maximized by carefully optimizing caching placement probabilities [14]. Moreover, the caching scheme in [11] was extended to the two-tier HetNet consisting of macro BSs and pico-BSs, where macro BSs adopt the MPC strategy and also take the influence of downloading the file from the core network into account, while pico-BSs could randomly cache the remaining files [15]. The hit probability was maximized by optimizing caching placement probabilities [15]. Moreover, for the multi-tier HetNet, optimal caching placement probabilities were derived to maximize the hit probability by considering the different parameters for different tiers, for example transmission power and received SIR threshold for the successful transmission [10]. For the multi-tier multi-antenna HetNet, each BS of every tiers is equipped with multiple antennas, and the hit probability was maximized by optimizing caching placement probabilities [16].

For D2D networks, the hit probability was maximized by optimizing caching placement probabilities and imposing the constraint that the same file cannot be cached at the nodes which are close to each other [17]. Furthermore, optimal caching placement probabilities was presented to maximize the throughput of D2D networks [18].

Fundamentally, the hit probability depends on two factors, one is caching placement probability that the requested file is cached at the SBS, and the other is the coverage probability that the file can be successfully transmitted to the served user. Furthermore, the successful transmission depends on SINR at the receiver. In order to meet the heavy traffic demand, ultra-dense networks are widely used, where small cells are densely deployed [19]-[21]. As spectrum resources are limited, interference in small-cell networks significantly degrades the performance of networks, for example, deteriorating SINR [20], [21]. Therefore, interference management plays a critical role for improving the performance of small-cell networks [20], [21]. In order to enhance the hit probability, the coverage probability need to be improved, i.e., enhancing SINR. Hence, joint interference management and caching strategy need to be considered. For single-input single-output (SISO) small cell networks, joint successive interference cancellation and caching strategy was considered for parallel transmission [7]. Furthermore, for multiple-input single-output (MISO) small cell networks, joint zero forcing (ZF) and probabilistic caching scheme was proposed to maximize the hit probability, where $\mathrm{ZF}$ based interference cancellation was exploited to suppress the intra-cluster interference [22]. However, the previous work$s$ only consider the scenarios of SISO or MISO networks, where only one single antenna is equipped at each user. In fact, nowadays users are normally equipped with multiple antennas. Deploying multiple antennas is capable of significantly improving system performance and is adopted as one key technology for 5G and beyond wireless networks [23], [24]. The previous works for joint interference management and caching strategy for SISO or MISO networks cannot exploit the advantage of multiple antennas at users.

For MIMO networks, interference alignment (IA) has been being widely proposed as a promising interference management scheme, which can effectively remove the interference and improve degrees of freedom (DoF) of networks [25]-[32].

Against this background, in this paper, we investigate joint interference management and caching placement for MIMO systems to enhance the hit probability. To the best knowledge of authors, it is the first time to investigate the joint interference management and caching placement for MIMO systems. Our main contributions are as following:

1) We propose one novel joint IA and probabilistic caching (JIA-ProbC) scheme for MIMO small-cell networks which consists of SBSs and users, and both of them are equipped with multiple antennas. Furthermore, multiple SBSs simultaneously transmit the requested files to intended users using the same frequency, which results in severe interference. For the MIMO system, the transceiver beamforming matrices of the IA scheme can be jointly designed to eliminate the more interference. Hence the MIMO system is capable of simultaneously supporting more users interference-free than both the MISO system with the ZF precoding scheme and the SISO system without interference management, which can enhance the coverage probability and further improve the hit probability.

2) Based on the IA scheme for MIMO small-cell networks, we use stochastic geometry to derive the maximum hit probability. Firstly, the theoretical coverage probability is analytically approximated by a closed-from expression. Furthermore, optimizing caching placement probabilities for maximizing the hit probability is formulated as a convex problem, which is effectively solved.

3) Numerical results show that the derived theoretical coverage probability is very close to the simulation results and is higher than that for ZF scheme. Moreover, our scheme of JIA-ProbC can achieve a performance gain up to $30 \%$ against the existing joint $\mathrm{ZF}$ and probabilistic caching (JZF-ProbC) scheme and performance gain up to $120 \%$ against the joint IA and MPC (JIA-MPC) 
scheme, respectively.

The rest of the paper is organized as follows. The system model is presented in Section II, while the joint IA and probabilistic caching scheme is presented in Section III. Specifically, the IA based interference cancellation scheme for MIMO small-cell networks is presented in Section III-A. In Section III-B, the performance metric of hit probability is discussed, and in Section III-C, the coverage probability and the optimal caching placement probability for maximizing the hit probability are presented. In Section IV numerical results are provided to demonstrate the performance of the proposed joint IA and probabilistic caching scheme in MIMO small-cell networks, while the conclusion is offered in Section V.

Notations: The unbolded letters represent scalars, the lowercase bold letters represent vectors and the uppercase bold letters represent the matrices. The operator $(\cdot)^{H}$ denotes Hermitian transpose of a vector or matrix, $\|\cdot\|$ is the standard Euclidean norm and $A \backslash B$ denotes a set in set $A$ while not in set $B$.

\section{SySTEM MODEL}

\section{A. System Configuration}

The system considered in this paper consists of one macro BS and one tier of SBSs. Furthermore, the location of SBSs and mobile users are modeled as independent HPPPs with intensity $\lambda_{B}$ and $\lambda_{U}$, respectively, and $\lambda_{U} \gg \lambda_{B}$ [22], denoted by $\Phi_{B}=\left\{\boldsymbol{d}_{k} \in \mathbb{R}^{2}, k \in \mathbb{N}^{+}\right\}$and $\Phi_{U}=\left\{\boldsymbol{u}_{k} \in \mathbb{R}^{2}, k \in \mathbb{N}^{+}\right\}$ [33]. And the set of SBSs is denoted by $\mathcal{B}=\left\{B_{i}, i \in \mathbb{N}^{+}\right\}$, where $B_{i}$ is the SBS located at $\boldsymbol{d}_{i}$. Moreover, each SBS and each user are equipped with $M$ and $N$ antennas, respectively.

The file library of the system contains $L$ files, and the size of each file is normalized to 1 [7], [8], [10]. The set of all files is denoted by $\mathcal{F}=\left\{F_{1}, F_{2}, \cdots, F_{L}\right\}$, where $F_{l}$ denotes the $l$-th most popular file. Every SBS is equipped with a cache of size $C$. Limited by the cache size, SBSs cannot cache all files, i.e, $C<L$. Furthermore, file $F_{l}$ is independently cached by every SBS with the placement probability $p_{l}$, and $\sum_{l=1}^{L} p_{l} \leq C$ [8], [10], [12], [13]. Moreover, users independently request file $F_{l}$ with the probability $q_{l}$, i.e. the popularity [8], which follows Zipf distribution with skewness parameter $\gamma$ [8].

Due to $\lambda_{U} \gg \lambda_{B}$, every SBS is considered to be always active and transmit signals in each time slot [22].

\section{B. Clustering Model}

In this paper, SBSs are grouped into clusters. Specifically, the plane is tessellated into $K$-th Voronoi cells [34], as shown in Fig. 1 . The $K$ SBSs $\left\{B_{1}, B_{2}, \cdots, B_{K}\right\}$ form a cluster, denoted as $\phi$. The $K$-th order Voronoi cell associated with $\left\{\boldsymbol{d}_{1}, \boldsymbol{d}_{2}, \cdots, \boldsymbol{d}_{K}\right\}$ is denoted as $\mathcal{V}_{K}\left\{\boldsymbol{d}_{1}, \boldsymbol{d}_{2}, \cdots, \boldsymbol{d}_{K}\right\}$ where all points are closer to $\left\{\boldsymbol{d}_{1}, \boldsymbol{d}_{2}, \cdots, \boldsymbol{d}_{K}\right\}$ than any other point of $\Phi_{B}$ [34], i.e.,

$$
\begin{aligned}
& \mathcal{V}_{K}\left\{\boldsymbol{d}_{1}, \boldsymbol{d}_{2}, \cdots, \boldsymbol{d}_{K}\right\}=\left\{\boldsymbol{d} \in \mathbb{R}^{2} \mid\right. \\
&\left.\cap_{k=1}^{K}\left\{\left\|\boldsymbol{d}-\boldsymbol{d}_{k}\right\| \leq\left\|\boldsymbol{d}-\boldsymbol{d}_{i}\right\|\right\}\right\},
\end{aligned}
$$

where $\forall \boldsymbol{d}_{i} \in \Phi_{B} \backslash\left\{\boldsymbol{d}_{1}, \boldsymbol{d}_{2}, \cdots, \boldsymbol{d}_{K}\right\}$.

For one $K$-th Voronoi cell, each SBS in the cluster can only serve one user in one time slot. Hence, $K$ users are simultaneously served by $K$ SBS using the same frequency in one time slot. Hence there is interference among the $K$ users. Furthermore, when the SBS caches the user's requested file and successfully transmits it to the user, the burden of the backhaul and the transmission latency is reduced and the performance of network can be improved [10]. Moreover, when one user requests a file, the closest SBS which caches the requested file within the cluster serves this user and transmits the requested file to this user, as this SBS normally provides the strongest strength of the signal at receiver among all SBSs caching the requested file [10], [11], [15]. If no SBS in the cluster caches the requested file, then macro BS will download the file over the backhaul and forward to the user [10].

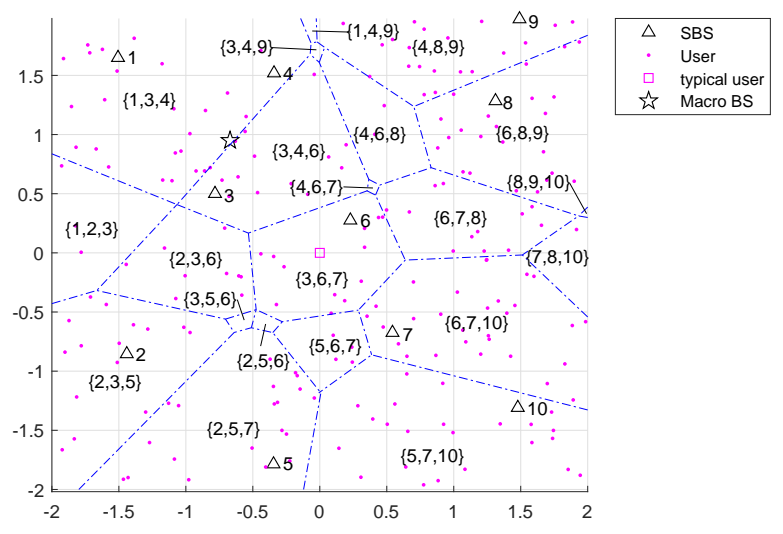

Fig. 1. The 3-th order Voronoi cells, where $\{a, b, c\}$ represents $\mathcal{V}_{3}\left\{\boldsymbol{d}_{a}, \boldsymbol{d}_{b}, \boldsymbol{d}_{c}\right\}$.

\section{Communication Model}

According to Slivnyak-Mecke theorem [35], it is sufficient to analyze system preformance by the typical user located at the origin [35]. Without loss of generality, SBSs can be indexed in an increasing order of distance between the typical user and SBSs, and the typical user is assumed in the $K$-th Voronoi cell $\mathcal{V}_{K}\left\{\boldsymbol{d}_{1}, \boldsymbol{d}_{2}, \cdots, \boldsymbol{d}_{K}\right\}$. Furthermore, the typical user is associated with the SBS $B_{k} \in \phi$ in one time slot.

It is assumed that a perfect knowledge of channel state information, which can be estimated by the sophisticated channel estimation method at receivers and fedback to transmitters [36], is available both transmitters and receivers.

The SBS $B_{i} \in \mathcal{B}$ transmits one data stream symbol $x_{i} \in \mathbb{C}$ with $\mathbb{E}\left|x_{i}\right|^{2}=1$ to the associated user in one time slot, with a precoding vector $\boldsymbol{v}_{i} \in \mathbb{C}^{M \times 1}$ and $\left\|\boldsymbol{v}_{i}\right\|^{2}=1$. The small-scale channel between the SBS $B_{i}$ and the typical user is denoted as $\mathbf{H}_{0 i} \in \mathbb{C}^{N \times M}$, where entries of $\mathbf{H}_{0 i}$ are drawn from $\mathcal{C N}(0,1)$. The received signal vector $\boldsymbol{y}_{0} \in \mathbb{C}^{N \times 1}$ at the typical user can be written as

$$
\begin{aligned}
\boldsymbol{y}_{0}= & \underbrace{r_{k}^{-\alpha / 2} \mathbf{H}_{0 k} \boldsymbol{v}_{k} x_{k}}_{\text {desired signal }}+\underbrace{\sum_{B_{i} \in \phi \backslash B_{k}} r_{i}^{-\alpha / 2} \mathbf{H}_{0 i} \boldsymbol{v}_{i} x_{i}}_{\text {intra-cluster interference }} \\
& +\underbrace{\sum_{B_{j} \in \mathcal{B} \backslash \phi} r_{j}^{-\alpha / 2} \mathbf{H}_{0 j} \boldsymbol{v}_{j} x_{j}}_{\text {inter-cluster interference }}+\boldsymbol{n}_{0},
\end{aligned}
$$


where $r_{i}$ is the distance between the SBS at $\boldsymbol{d}_{i}$ and the typical user, i.e. $r_{i}=\left\|\boldsymbol{d}_{i}\right\|$, and $\alpha$ is path loss exponent. $r_{k}^{-\alpha / 2} \mathbf{H}_{0 k} \boldsymbol{v}_{k} x_{k}$ denotes the desired signal from the associated SBS at $\boldsymbol{d}_{k}$, while $\sum_{B_{i} \in \phi \backslash B_{k}} r_{i}^{-\alpha / 2} \mathbf{H}_{0 i} \boldsymbol{v}_{i} x_{i}$ denotes the intracluster interference by other SBSs in the cluster $\phi$, and $\sum_{B_{j} \in \mathcal{B} \backslash \phi} r_{j}^{-\alpha / 2} \mathbf{H}_{0 j} \boldsymbol{v}_{j} x_{j}$ denotes the inter-cluster interference by SBSs from other clusters. $\boldsymbol{n}_{0}$ denotes noise and is ignored in the following analysis as interference dominates the received signal [22].

After applying the decoding vector $\boldsymbol{u}_{k} \in \mathbb{C}^{N \times 1}$ with $\left\|\boldsymbol{u}_{k}\right\|^{2}=1$, the typical user obtains decision symbol $\hat{y} \in \mathbb{C}$,

$$
\begin{aligned}
\hat{y}_{0}= & \boldsymbol{u}_{k}^{H} \boldsymbol{y}_{0} \\
= & \underbrace{r_{k}^{-\alpha / 2} \boldsymbol{u}_{k}^{H} \mathbf{H}_{0 k} \boldsymbol{v}_{k} x_{k}}_{\text {desired signal }}+\underbrace{\sum_{B_{i} \in \phi \backslash B_{k}} r_{i}^{-\alpha / 2} \boldsymbol{u}_{k}^{H} \mathbf{H}_{0 i} \boldsymbol{v}_{i} x_{i}}_{\text {intra-cluster interference }} \\
& +\underbrace{\sum_{B_{j} \in \mathcal{B} \backslash \phi} r_{j}^{-\alpha / 2} \boldsymbol{u}_{k}^{H} \mathbf{H}_{0 j} \boldsymbol{v}_{j} x_{j}}_{\text {inter-cluster interference }} .
\end{aligned}
$$

For this MIMO interference channel networks, by jointly designing precoding vectors and decoding vectors $\left\{\boldsymbol{v}_{i}, \boldsymbol{u}_{i}\right\}_{1 \leq i \leq K}$, the intra-cluster interference may be eliminated [25], [37]. In this paper, IA schemes are exploited to eliminate the intra-cluster interference.

\section{Joint Interference ALIGNMENT AND PROBABILISTIC CACHING}

\section{A. IA Based Interference Cancellation}

As only one single data stream is transmitted by each $\mathrm{SBS}$, in order to eliminate the intra-cluster interference, the following IA conditions must be satisfied [26]

$$
\begin{aligned}
\boldsymbol{u}_{k}^{H} \mathbf{H}_{0 i} \boldsymbol{v}_{i} & =0, & i \in \phi \backslash B_{k} \\
\boldsymbol{u}_{k}^{H} \mathbf{H}_{0 k} \boldsymbol{v}_{k} & \neq 0, & k \in \phi
\end{aligned}
$$

where equation (4) is used to eliminate the intra-cluster interference, while equation (5) is used to retain the desired signal. As the channel is generic, when (4) is satisfied with non-zero $\left\{\boldsymbol{v}_{i}, \boldsymbol{u}_{i}\right\}_{1 \leq i \leq K}$, (5) is satisfied automatically [27].

Furthermore, the feasibility condition of IA can be written as [26]

$$
K \leq M+N-1 \text {. }
$$

In this paper, $K$ is set to be $M+N-1$ to maximize the number of users served by SBSs from the cluster simultaneously.

Once the IA feasibility condition (6) is satisfied, there exist appropriate precoding vectors and decoding vectors $\left\{\boldsymbol{v}_{i}, \boldsymbol{u}_{i}\right\}_{1 \leq i \leq K}$ which meet IA equations (4) [26] and can be obtained by using the existing methods [27], [28]. In this case, the intra-cluster interference can be completely eliminated, and the decision symbol $\hat{y}_{0}$ is given by

$$
\hat{y}_{0}=\underbrace{r_{k}^{-\alpha / 2} \boldsymbol{u}_{k}^{H} \mathbf{H}_{0 k} \boldsymbol{v}_{k} x_{k}}_{\text {desired signal }}+\underbrace{\sum_{B_{j} \in \mathcal{B} \backslash \phi} r_{j}^{-\alpha / 2} \boldsymbol{u}_{k}^{H} \mathbf{H}_{0 j} \boldsymbol{v}_{j} x_{j}}_{\text {inter-interference }} .
$$

Consequently, the desired signal of the typical user received is only affected by the inter-cluster interference, and the $\mathrm{SIR}_{k}$ of the typical user can be written as

$$
\mathrm{SIR}_{k}=\frac{r_{k}^{-\alpha} g_{k}}{\sum_{B_{j} \in \mathcal{B} \backslash \phi} r_{j}^{-\alpha} g_{j}},
$$

where $g_{i}=\left|\boldsymbol{u}_{k}^{H} \mathbf{H}_{0 i} \boldsymbol{v}_{i}\right|^{2}, \forall B_{i} \in \mathcal{B}$.

Based on the IA scheme, optimal caching placement probabilities need to be derived to maximize the hit probability.

\section{B. Hit Probability}

The hit probability $P_{\text {hit }}$ is defined as the probability that a SBS in the cluster caches the typical user's requested file and successfully transmits the file to the typical user over the wireless channel [10]. The higher the hit probability, the less the backhaul link burden [10] as well as the lower the delay [38]. $P_{\text {hit }}$ can be written as [10], [22]

$$
P_{\text {hit }}=\sum_{l=1}^{L} q_{l} P_{\text {hit }}^{l}
$$

where $P_{\text {hit }}^{l}$ denotes the probability that the typical user requests file $F_{l}$ and file $F_{l}$ is successfully transmitted to the typical user, which can be formulated as [22]

$$
P_{\mathrm{hit}}^{l}=\sum_{k=1}^{K} p_{l}\left(1-p_{l}\right)^{k-1} P_{\mathrm{cov}}^{k},
$$

where $p_{l}\left(1-p_{l}\right)^{k-1}$ denotes the probability that the closest SBS which caches the requested file $F_{l}$ in the cluster is the $k$-th closest SBS $B_{k}$ to the typical user. Furthermore, $P_{\mathrm{cov}}^{k}$, referred as coverage probability [22], denotes the probability that $\mathrm{SIR}_{k}$ between $B_{k}$ and the typical user is greater than the SIR threshold $\theta$, which can be written as [10], [22]

$$
P_{\mathrm{cov}}^{k}=\mathbb{P}\left(\operatorname{SIR}_{k} \geq \theta\right),
$$

which indicates the probability that the file can be successfully transmitted to the typical user by $B_{k}$.

Upon substituting (10) into (9), we have

$$
P_{\text {hit }}=\sum_{l=1}^{L} q_{l} \sum_{k=1}^{K} p_{l}\left(1-p_{l}\right)^{k-1} P_{\mathrm{cov}}^{k}
$$

\section{Approximate Optimal Probabilistic Caching}

Our design goal is to maximize the hit probability $P_{\text {hit }}$ by determining the optimal caching placement probabilities $\left\{p_{l}\right\}$, and the optimization problem can be formulated as [22]

$$
\begin{aligned}
P 1: \max _{\left\{p_{l}\right\}} & \sum_{l=1}^{L} q_{l} \sum_{k=1}^{K} p_{l}\left(1-p_{l}\right)^{k-1} P_{\mathrm{cov}}^{k} \\
\text { s.t } \quad & \sum_{l=1}^{L} p_{l} \leq C \\
& C \leq L \\
& 0 \leq p_{l} \leq 1, \forall l
\end{aligned}
$$


For the problem $P 1, P_{\text {cov }}^{k}$ can be expressed as [22]

$$
\begin{aligned}
P_{\mathrm{cov}}^{k} & =\mathbb{P}\left(\operatorname{SIR}_{k} \geq \theta\right) \\
& =\mathbb{P}\left(\frac{r_{k}^{-\alpha} g_{k}}{\sum_{B_{j} \in \mathcal{B} \backslash \phi} r_{j}^{-\alpha} g_{j}} \geq \theta\right) \\
& =\mathbb{P}\left(g_{k} \geq \theta r_{k}^{\alpha} I\right) \\
& =\mathbb{E}\left[e^{-\theta r_{k}^{\alpha} I} \mid r_{k}, I\right]
\end{aligned}
$$

where $I=\sum_{B_{j} \in \mathcal{B} \backslash \phi} r_{j}^{-\alpha} g_{j}$.

As $\left\{\boldsymbol{u}_{k}, \boldsymbol{v}_{k}\right\}$ are only determined by the intra-cluster interference channels within one cluster, $\left\{\boldsymbol{u}_{k}, \boldsymbol{v}_{k}\right\}$ are independent with the direct channel $\mathbf{H}_{0 k}$ and inter-cluster interference channels $\left\{\mathbf{H}_{0 j}\right\}_{B_{j} \in \mathcal{B} \backslash \phi}$. As entries of both $\mathbf{H}_{0 k}$ and $\left\{\mathbf{H}_{0 j}\right\}_{B_{j} \in \mathcal{B} \backslash \phi}$ are drawn from complex Gaussian distribution, and $\boldsymbol{u}_{k}$ and $\left\{\boldsymbol{v}_{j}\right\}_{B_{j} \in \mathcal{B} \backslash \phi}$ are independent with both $\mathbf{H}_{0 k}$ and $\left\{\mathbf{H}_{0 j}\right\}_{B_{j} \in \mathcal{B} \backslash \phi}, g_{j}$ follows the exponential distribution with parameter one [39], [40], i.e, $g_{j} \sim \exp (1), \forall B_{j} \in \mathcal{B}$. Furthermore, according to [22, Theorem 2] ${ }^{1}, P_{\text {cov }}^{k}$ can be approximated as [22]

$$
P_{\mathrm{cov}}^{k} \simeq \frac{1}{\left[1+\theta^{2 / \alpha} \sqrt{\frac{k}{K}} \mathcal{G}\left(\frac{\sqrt{K} \theta^{-2 / \alpha}}{\sqrt{k}}\right)\right]^{k}},
$$

where $\mathcal{G}(x)=\int_{x}^{\infty} \frac{1}{1+v^{\alpha / 2}} d v$.

According to (18), $P 1$ can be approximately formulated as

$$
\begin{aligned}
& P 2: \min _{\left\{p_{l}\right\}} \quad-\sum_{l=1}^{L} q_{l} \sum_{k=1}^{K} p_{l}\left(1-p_{l}\right)^{k-1} \\
& {\left[1+\theta^{2 / \alpha} \sqrt{\frac{k}{K}} \mathcal{G}\left(\frac{\sqrt{K} \theta^{-2 / \alpha}}{\sqrt{k}}\right)\right]^{-k} } \\
& \text { s.t } \quad \sum_{l=1}^{L} p_{l} \leq C \\
& C \leq L \\
& 0 \leq p_{l} \leq 1, \forall l
\end{aligned}
$$

According to [22, Lemma 3], the problem $P 2$ is a convex problem, and the Lagrange function is given by [22]

$$
\begin{aligned}
L\left(p_{1}, p_{2}, \cdots, p_{L}, u\right)= & -\sum_{l=1}^{L} q_{l} \sum_{k=1}^{K} p_{l}\left(1-p_{l}\right)^{k-1} P_{\mathrm{cov}}^{k} \\
& +u\left(\sum_{l=1}^{L} p_{l}-C\right),
\end{aligned}
$$

where $u$ is the Lagrangian multiplier associated with the constraint (19b). Furthermore, KKT conditions are given by

\footnotetext{
${ }^{1}$ In [22], the ZF scheme is adopted to cancel interference, where each user is equipped one single antenna. Each SBS transmits one single data stream to its served user, where the effective channel gain of the desired signal follows Gamma distribution $\Gamma(M-K+1,1)$. However, when $M=K$, the distribution of the effective channel gain of the desired signal is reduced to exponential distribution $\exp (1)$.
}

[22]

$$
\begin{aligned}
-q_{l} \sum_{k=1}^{K}\left(1-k p_{l}^{*}\right)\left(1-p_{l}^{*}\right)^{k-2} P_{\mathrm{cov}}^{k}+u^{*} & =0, \\
\sum_{l=1}^{L} p_{l}^{*}-C & =0 .
\end{aligned}
$$

Moreover, the optimal caching placement probabilities of probabilistic caching can be obtained as [22]

$$
p_{l}^{*}= \begin{cases}1, & \text { if } q_{l} \geq \frac{u^{*}}{P_{\mathrm{cov}}^{1}-P_{\mathrm{cov}}^{2}} \\ w_{l}\left(u^{*}\right), & \text { if } \frac{u^{*}}{\sum_{k=1}^{K} P_{\mathrm{cov}}^{k}}<q_{l}<\frac{u^{*}}{P_{\mathrm{cov}}-P_{\mathrm{cov}}^{2}} \\ 0, & \text { if } q_{l} \leq \frac{u^{*}}{\sum_{k=1}^{K} P_{\mathrm{cov}}^{k}}\end{cases}
$$

with the constraint $\sum_{l=1}^{L} p_{l}^{*}-C=0$. Furthermore, $w_{l}\left(u^{*}\right)$ is a nonnegative real root of equation (21) [22]. Moreover, the optimal $u^{*}$ can be determined by the bisection method. From (23), we can see that the caching placement probability of the file increases with the increase of its popularity. Furthermore, when the popularity of the file is higher than $\frac{u^{*}}{P^{1}-P_{2 v}^{2}}$, the caching placement probability is 1 , while for a popularity lower than $\frac{u^{*}}{\sum_{k=1}^{K} P_{\mathrm{cov}}^{k}}$, the caching placement probability is 0 .

\section{Numerical Results}

In this section, we demonstrate the performance of the proposed JIA-ProbC scheme in MIMO small-cell networks. In the following simulations, the area is $4000 \mathrm{~m} \times 4000 \mathrm{~m}$, SBS intensity is $\lambda_{B}=2 \times 10^{-5}$, and path loss exponent is $\alpha=4$. The popularity of files follows Zipf distribution with the skewness parameter $\gamma=0.9$. The size of the file library is $L=100$, and the cache size is $C=10$ [22].Furthermore, the JIA-MPC scheme is used as the benchmark, where the MPC caching strategy is employed to all SBSs, i.e., all SBSs only cache the most popular file until the cache space is full.

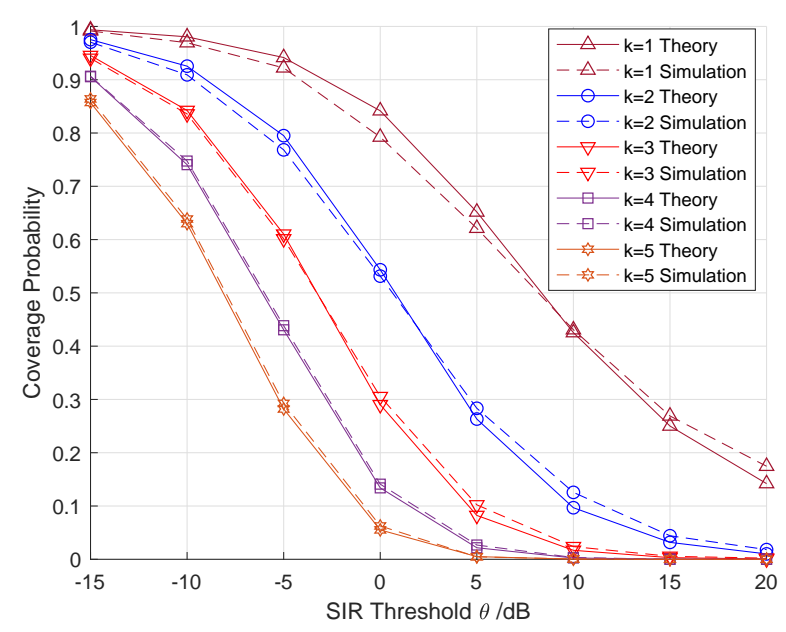

Fig. 2. The coverage probability for both theories and simulations with $M=4, N=2$ and $K=5$ in IA scheme.

In Fig. 2, the coverage probability versus the SIR threshold is plotted for both theories of (18) and simulations with $M=$ $4, N=2$ and $K=5$, where $k$ indicates that the typical user 
requests file from the $k$-th closest SBS. As seen from Fig. 2 , the theoretical results obtained by (18) match the result by simulations very well. Moreover, the coverage probability decreases with the increasing of $k$, as the strength of desired signal decreases with the increasing of the distance between the typical user and serving SBS, resulting the decreasing of SIR at the typical user.

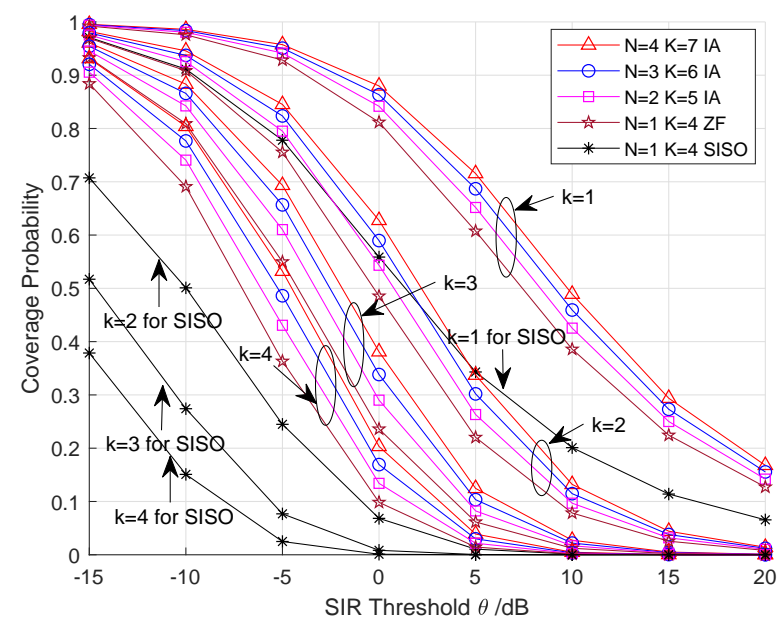

Fig. 3. The theoretical coverage probability from the $k$-th closest SBS.

In Fig. 3, the theoretical coverage probability versus the SIR threshold is plotted for the different $K$-th order Voronoi cells with $M=4, N=1,2,3$ and 4 , as well as $K=4,5,6$ and 7 and the SISO system with $M=1$ and $N=1$, where $k$ indicates that the typical user requests file from the $k$ th closest SBS. For $N=1$ and $K=4$, the IA scheme is reduced to the ZF scheme for interference cancellation, while for the other configurations, the IA scheme is used. As we can see from Fig. 3, when the $k$-th SBS serves the typical user, the coverage probability increases with the increasing of the number of antennas $N$ at the typical user. The reason is that the increase of the number of antennas $N$ results in the increase of the number of SBSs $K$ in the cluster. Therefore, the more interference can be included as intra-cluster interference and can cancel by IA schemes. Moreover, for a given the number of SBSs $K$ in the cluster, the coverage probability decreases with the increasing of $k$. This phenomenon is the same as observed in Fig. 2. Moreover, for the SISO system, $K$-th Voronoi cells is also used to form the cluster and each user only obtains the requested file from the closest SBS having the required file in the cluster. As we can see the SISO system achieves the worst performance, which is due to no interference is eliminated.

In Fig. 4, the hit probability versus the SIR threshold is plotted for $M=4, N=1,2,3$ and 4 , as well as $K=$ 4, 5, 6 and 7 and the SISO system with $M=1$ and $N=1$. As we can see from Fig. 4, the hit probability increases with the increase of the number of antennas $N$. The reason is that the hit probability increases with the increase of the coverage probability according to the equation (12), and as seen from Fig. 3 the coverage probability increases with the increase of the number of antennas $N$. Furthermore, the performance gain of the $N=4$ in the proposed JIA-ProbC scheme against

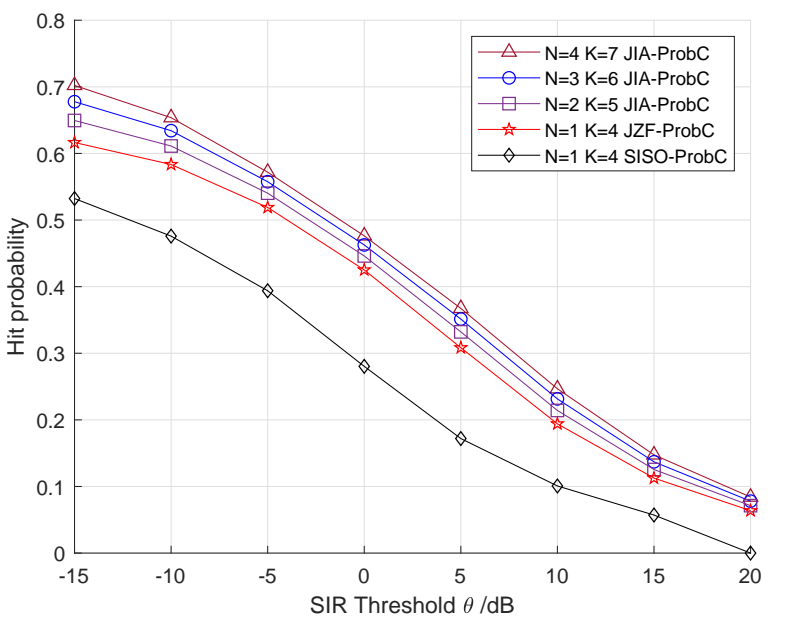

Fig. 4. The hit probability versus SIR threshold for different $K$-th Voronoi cells.

$N=1$ in the JZF-ProbC scheme varies from $14 \%$ to $30 \%$, when SIR threshold changes from $\theta=-15 \mathrm{~dB}$ to $\theta=15 \mathrm{~dB}$. Moreover, the SISO system achieves the worst performance. Therefore, interference management can remarkably enhance the performance by employing multiple antennas.

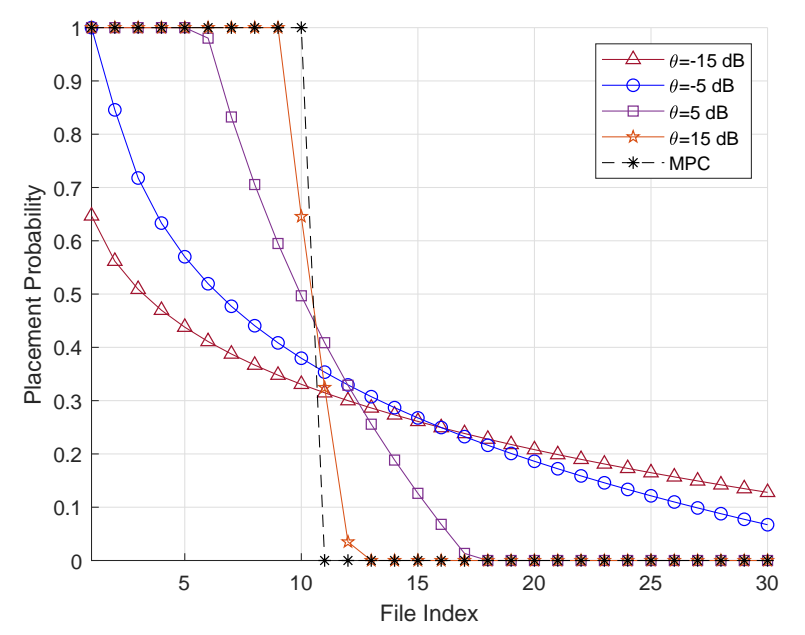

Fig. 5. Caching placement probabilities versus the file index under different SIR thresholds for both the JIA-ProbC scheme and the JIA-MPC scheme.

In Fig. 5, the caching placement probabilities versus the file index is plotted under different SIR thresholds for both the proposed JIA-ProbC scheme and the JIA-MPC scheme with $M=4, N=2$ and $K=5$. As seen from Fig. 5, for the JIAMPC scheme, the first 10 files are cached at each SBS with probability one, while the remaining files are not cached at any SBS. The reason is that for the JIA-MPC scheme only the files with highest popularity are cached which is independent of SIR threshold. Furthermore, as seen from Fig. 5, for the JIAProbC scheme, the caching placement probabilities of files with high popularity increases with the increase of the SIR threshold, while the caching placement probabilities of files with low popularity increases with the decrease of the SIR threshold. The reason is that under the higher SIR threshold, only the SBSs closer to the user can provide the desired 
signal with enough strength. Hence, the user can only obtain the requested file from those closer SBSs with high SIR. In order to improve the hit probability, the SBS has to cache the files with higher popularity, which are requested by the user with the high possibility. On the other hand, under the lower SIR threshold, even the SBS farther from the user may still be able to provide the desired signal with enough strength. Even if the requested files are not cached in the closer SBS, the requested files can be obtained from the farther SBS. Hence, SBSs in the cluster cooperatively cache more number of different files to satisfy the various file requests. In other words, caching placement probabilities of the files with high popularity can appropriately be reduced so that the files with low popularity can be available in the cluster. Moreover, as seen from Fig. 5, under the high SIR threshold the caching placement probabilities for the JIA-ProbC scheme are similar to those for the JIA-MPC scheme. The reason is that with the increase of SIR threshold the caching placement probabilities of first 10 files tend to be one, while those of the remaining files tend to be zero, which converges to caching placement probabilities for the JIA-MPC scheme.

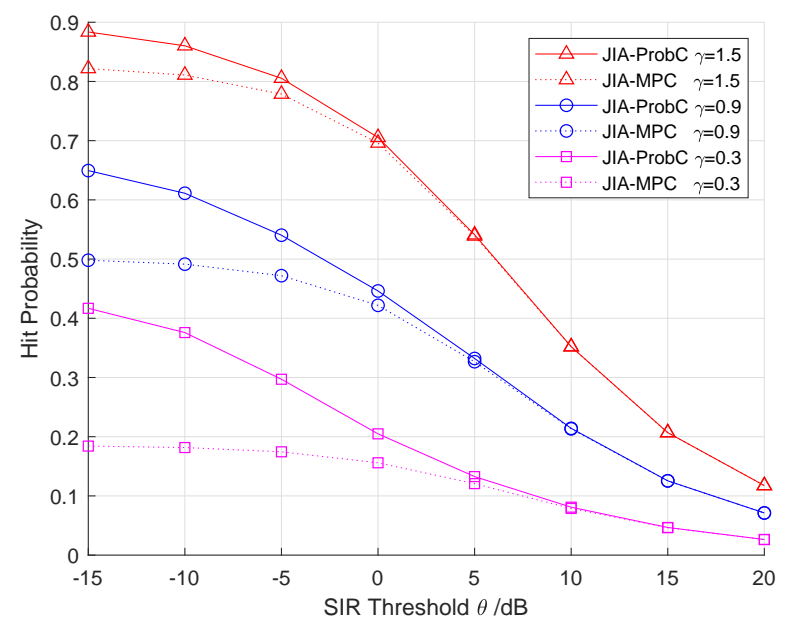

Fig. 6. The hit probability versus SIR threshold for both the JIA-ProbC scheme and the JIA-MPC scheme.

In Fig. 6, the hit probability is plotted versus the SIR threshold for Zipf skewness parameter $\gamma=0.3,0.9$ and 1.5 with $M=4, N=2$ and $K=5$. As seen from Fig. 6 , the hit probability decreases with the increase of SIR threshold. For a given Zipf skewness parameter $\gamma$, the gap between the JIA-ProbC scheme and the JIA-MPC scheme increases with the decrease of SIR threshold. The reason is that under the low SIR threshold SBSs in the cluster cooperatively cache more different files for the JIA-ProbC scheme, so that to satisfy various the file requests, as seen from Fig. 5. Therefore, under the lower SIR threshold using the JIA-ProbC scheme can provides a greater chance for the user to obtain files with low popularity than that using the JIA-MPC scheme, which improves the hit probability. Specifically, the performance gain of the JIA-ProbC scheme against JIA-MPC is up to $120 \%$ under $\theta=-15 \mathrm{~dB}$ and $\gamma=0.3$. However, under high SIR threshold the caching placement probabilities for the JIAProbC scheme is similar to those for the JIA-MPC scheme, as seen from Fig. 5, so that the hit probabilities for both the JIA-ProbC scheme and the JIA-MPC scheme are similar. Moreover, as seen from Fig. 6, the gap between the JIAProbC scheme and the JIA-MPC scheme decreases with the increase of Zipf skewness parameter $\gamma$. The reason is that the higher Zipf skewness parameter indicates that the first 10 files have higher popularities than those for the lower Zipf skewness parameter, which means that the first 10 files may be requested with higher probability. In this case, the caching placement probabilities of the first 10 files are increased, while the caching placement probabilities of the remaining files with low popularity are reduced. Hence, for high Zipf skewness parameters, the caching placement probabilities for both the JIA-ProbC scheme and the JIA-MPC scheme are similar. Therefore, the gap between the JIA-ProbC scheme and the JIA-MPC scheme decreases.

\section{CONCLUSION}

In this paper, one joint interference alignment and probabilistic caching scheme for MIMO small-cell networks is proposed. By employing IA scheme for MIMO IC networks, more interference can be canceled, which improve the coverage probability, and further enhance the hit probability. The coverage probability and caching placement probability are analytically presented based on stochastic geometry. Numerical results show that our proposed the JIA-ProbC scheme outperforms the existing JZF-ProbC scheme as well as the JIA-MPC scheme.

\section{REFERENCES}

[1] Y. Zhang, M. S. Hossain, A. Ghoneim, and M. Guizani, "Cocme: Content-oriented caching on the mobile edge for wireless communications," IEEE Wireless Communications, vol. 26, no. 3, pp. 26-31, 2019.

[2] X. Wang, M. Chen, T. Taleb, A. Ksentini, and V. C. M. Leung, "Cache in the air: Exploiting content caching and delivery techniques for 5G systems," IEEE Communications Magazine, vol. 52, no. 2, pp. 131-139, 2014.

[3] D. Liu, B. Chen, C. Yang, and A. F. Molisch, "Caching at the wireless edge: Design aspects, challenges, and future directions," IEEE Communications Magazine, vol. 54, no. 9, pp. 22-28, 2016.

[4] E. Bastug, M. Bennis, and M. Debbah, "Living on the edge: The role of proactive caching in $5 \mathrm{G}$ wireless networks," IEEE Communications Magazine, vol. 52, no. 8, pp. 82-89, 2014.

[5] K. Shanmugam, N. Golrezaei, A. G. Dimakis, A. F. Molisch, and G. Caire, "Femtocaching: Wireless content delivery through distributed caching helpers," IEEE Transactions on Information Theory, vol. 59, no. 12 , pp. 8402-8413, 2013.

[6] C. Yang, Y. Yao, Z. Chen, and B. Xia, "Analysis on cache-enabled wireless heterogeneous networks," IEEE Transactions on Wireless Communications, vol. 15, no. 1, pp. 131-145, 2016.

[7] Z. Chen, J. Lee, T. Q. S. Quek, and M. Kountouris, "Cooperative caching and transmission design in cluster-centric small cell networks," IEEE Transactions on Wireless Communications, vol. 16, no. 5, pp. 34013415, 2017.

[8] B. Blaszczyszyn and A. Giovanidis, "Optimal geographic caching in cellular networks," in 2015 IEEE International Conference on Communications (ICC), 2015, pp. 3358-3363.

[9] Y. Chen, M. Ding, J. Li, Z. Lin, G. Mao, and L. Hanzo, "Probabilistic small-cell caching: Performance analysis and optimization," IEEE Transactions on Vehicular Technology, vol. 66, no. 5, pp. 4341-4354, 2017.

[10] J. Wen, K. Huang, S. Yang, and V. O. K. Li, "Cache-enabled heterogeneous cellular networks: Optimal tier-level content placement," IEEE Transactions on Wireless Communications, vol. 16, no. 9, pp. 59395952, 2017 
[11] Y. Cui, D. Jiang, and Y. Wu, "Analysis and optimization of caching and multicasting in large-scale cache-enabled wireless networks," IEEE Transactions on Wireless Communications, vol. 15, no. 7, pp. 51015112, 2016.

[12] S. H. Chae and W. Choi, "Caching placement in stochastic wireless caching helper networks: Channel selection diversity via caching," IEEE Transactions on Wireless Communications, vol. 15, no. 10, pp. 66266637, 2016.

[13] S. H. Chae, T. Q. S. Quek, and W. Choi, "Content placement for wireless cooperative caching helpers: A tradeoff between cooperative gain and content diversity gain," IEEE Transactions on Wireless Communications, vol. 16, no. 10, pp. 6795-6807, 2017.

[14] S. Zhang, W. Sun, and J. Liu, "Spatially cooperative caching and optimization for heterogeneous network," IEEE Transactions on Vehicular Technology, vol. 68, no. 11, pp. 11260-11270, 2019.

[15] Y. Cui and D. Jiang, "Analysis and optimization of caching and multicasting in large-scale cache-enabled heterogeneous wireless networks," IEEE Transactions on Wireless Communications, vol. 16, no. 1, pp. 250 264, 2017

[16] S. Kuang, X. Liu, and N. Liu, "Analysis and optimization of random caching in $K$-Tier multi-antenna multi-user HetNets," IEEE Transactions on Communications, vol. 67, no. 8, pp. 5721-5735, 2019.

[17] D. Malak, M. Al-Shalash, and J. G. Andrews, "Spatially correlated content caching for device-to-device communications," IEEE Transactions on Wireless Communications, vol. 17, no. 1, pp. 56-70, 2018.

[18] N. Zhao, X. Liu, Y. Chen, S. Zhang, Z. Li, B. Chen, and M. Alouini, "Caching D2D connections in small-cell networks," IEEE Transactions on Vehicular Technology, vol. 67, no. 12, pp. 12 326-12 338, 2018.

[19] D. Lpez-Prez, M. Ding, H. Claussen, and A. H. Jafari, "Towards $1 \mathrm{Gbps} / \mathrm{UE}$ in cellular systems: Understanding ultra-dense small cell deployments," IEEE Communications Surveys Tutorials, vol. 17, no. 4, pp. 2078-2101, 2015.

[20] M. Kamel, W. Hamouda, and A. Youssef, "Ultra-dense networks: A survey," IEEE Communications Surveys Tutorials, vol. 18, no. 4, pp. 2522-2545, 2016.

[21] J. Liu, M. Sheng, L. Liu, and J. Li, "Interference management in ultradense networks: Challenges and approaches," IEEE Network, vol. 31, no. 6, pp. 70-77, 2017.

[22] X. Xu and M. Tao, "Modeling, analysis, and optimization of caching in multi-antenna small-cell networks," IEEE Transactions on Wireless Communications, vol. 18, no. 11, pp. 5454-5469, 2019.

[23] J. Zhang, E. Bjrnson, M. Matthaiou, D. W. K. Ng, H. Yang, and D. J. Love, "Prospective multiple antenna technologies for beyond 5G," IEEE Journal on Selected Areas in Communications, vol. 38, no. 8, pp. 16371660, 2020.

[24] V. W. Wong, R. Schober, D. W. K. Ng, and L. Wang, Key technologies for $5 G$ wireless systems. U.K.: Cambridge University press, 2017.

[25] V. R. Cadambe and S. A. Jafar, "Interference alignment and degrees of freedom of the K-User interference channel," IEEE Transactions on Information Theory, vol. 54, no. 8, pp. 3425-3441, 2008.

[26] C. M. Yetis, T. Gou, S. A. Jafar, and A. H. Kayran, "On feasibility of interference alignment in MIMO interference networks," IEEE Transactions on Signal Processing, vol. 58, no. 9, pp. 4771-4782, 2010.

[27] K. Gomadam, V. R. Cadambe, and S. A. Jafar, "A distributed numerica approach to interference alignment and applications to wireless interference networks," IEEE Transactions on Information Theory, vol. 57, no. 6, pp. 3309-3322, 2011.

[28] D. S. Papailiopoulos and A. G. Dimakis, "Interference alignment as a rank constrained rank minimization," IEEE Transactions on Signal Processing, vol. 60, no. 8, pp. 4278-4288, 2012.

[29] W. Liu, J. Sun, J. Li, and Y. Ma, "Interference alignment for MIMO downlink multicell networks," IEEE Transactions on Vehicular Technology, vol. 65, no. 8, pp. 6159-6167, 2016.

[30] W. Liu, J. Cai, J. Li, and M. Sheng, "Interference alignment with finite extensions in partially connected networks," IEEE Transactions on Communications, vol. 65, no. 2, pp. 851-862, 2017.

[31] W. Liu, Y. Han, J. Li, and Y. Ma, "Topological interference management with transmitter cooperation for MIMO interference channels," IEEE Transactions on Vehicular Technology, vol. 67, no. 11, pp. 10564 $10573,2018$.

[32] W. Liu, Q. Shi, and J. Li, "On the feasibility of interference alignment with finite channel extensions for MIMO interference broadcast channels with common messages," IEEE Transactions on Wireless Communications, vol. 18, no. 10, pp. 4915-4926, 2019.

[33] H. ElSawy, E. Hossain, and M. Haenggi, "Stochastic geometry for modeling, analysis, and design of multi-tier and cognitive cellular wireless networks: A survey," IEEE Communications Surveys Tutorials, vol. 15, no. 3, pp. 996-1019, 2013.

[34] N. Lee, D. Morales-Jimenez, A. Lozano, and R. W. Heath, "Spectral efficiency of dynamic coordinated beamforming: A stochastic geometry approach," IEEE Transactions on Wireless Communications, vol. 14, no. 1, pp. 230-241, 2015.

[35] M. Haenggi, Stochastic Geometry for Wireless Networks. Cambridge: U.K.: Cambridge Univ. Press, 2012.

[36] S. Mosleh, J. D. Ashdown, J. D. Matyjas, M. J. Medley, J. Zhang, and L. Liu, "Interference alignment for downlink multi-cell lte-advanced systems with limited feedback," IEEE Transactions on Wireless Communications, vol. 15, no. 12, pp. 8107-8121, 2016.

[37] H. Sung, S.-H. Park, K.-J. Lee, and I. Lee, "Linear precoder designs for k-user interference channels," IEEE Transactions on Wireless Communications, vol. 9, no. 1, pp. 291-301, 2010.

[38] G. Zhang, T. Q. S. Quek, M. Kountouris, A. Huang, and H. Shan, "Fundamentals of heterogeneous backhaul design-analysis and optimization," IEEE Transactions on Communications, vol. 64, no. 2, pp. 876-889, 2016.

[39] T. M. Nguyen, Y. Jeong, T. Q. S. Quek, W. P. Tay, and H. Shin, "Interference alignment in a poisson field of MIMO femtocells," IEEE Transactions on Wireless Communications, vol. 12, no. 6, pp. 2633 2645, 2013.

[40] Y. Luo, T. Ratnarajah, J. Xue, and F. A. Khan, "Interference alignment in two-tier randomly distributed heterogeneous wireless networks using stochastic geometry approach," IEEE Systems Journal, vol. 12, no. 3, pp. 2238-2249, 2018. 